\title{
THE PEP INSTRUMENTATION AND CONTROL SYSTEM*)
}

Robert Melen

SLAC-PUB - 2553

June 1980

(I)

Stanford Linear Accelerator Center, Stanford University, Stanford, CA 94305

\begin{abstract}
This paper describes the operating tharacteristics of the primary components that form the pep Instrumentation anc Control System. Descriptions are provided for the computer control system, beam monitors, and other support systems.
\end{abstract}

\section{INTRODUCTION}

This paper will describe the operation and performance of the main components of the PEP Instrumentation and Control (IGC) System. The PEP, Ing has been operational since April, 1980, so all of the systems described here are operational except where explicitly stated.

Most of the IEC system equipment for PEP is distributed in six sunport areas (See Fig. 1), equally spaced around the 2200 meter circumference ring, and a seventh area near the entry point of the injection lines. The placement of electronic equipment in the ring tunnel was minimized so a]l heamline sensors and control elements are hardwired to the nearest suppore arca. All relevent control and monitoring signals associated with the system are either sent through computer links or through a relatively small hardwired cable plant to the PEP Control Room (PCR) which is located in the same building as the Region 8 support area. All of the normal ring control and monizoring operations are conducted from the PCR main console arca shown in

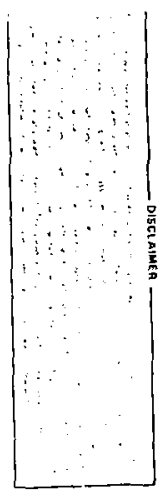
Fig. 2 .

Section 2 of this paper describes the system architccture, interface hardware design and applications support sortware associated with the computer control system. Section 3 describes the computer control and monitering of the power supply, RF and vacuum systems. Section 4 describes the operation of $t$ he beam monitors, and Section 5 provides a brief description of the timing, communications, and personnel protection systers.

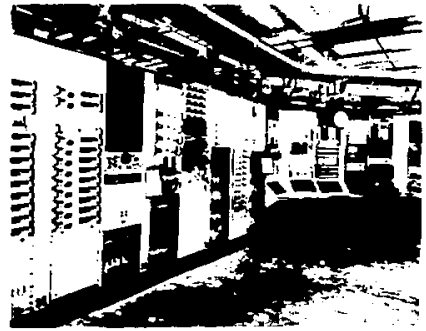

Fig. 1. A photograph of a typical PEP Instrumentation and Control equipment area.

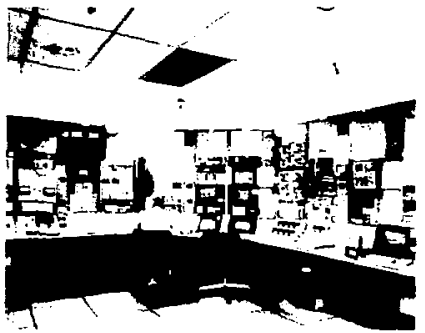

Fig. 2. The PFP main console area located in the PEP Control Room (PCR).

\footnotetext{
* Work supported by the Department of Energy, cantract DE-AC05-765F00515. (Invited talk presented at the XIth International Conference on High Energy Accelerators, CERN, Geneva, Switzerland, July 7-11, 1980.)
} 


\section{THE COMPUTER CONTROL SYSTEM}

\subsection{System Architecture}

A block diagram of the computer control system is shown in Fig. 3 . It contains a network of 10 ModComp computers and one Digital Equipment Corp. (DEC) PDP $11 / 780$ (VAX) computer. The MCIV central computer is attached to a single operator control console, and is connected via high speed (500 kilobaud) serial links to 9 MCII remote computers as well as the MCIV central secondary computer. The MCII remote computers are interfaced to a total of 50 CAMAC crates via high speed ( 1 mega-baud) serial links based on Synchro-

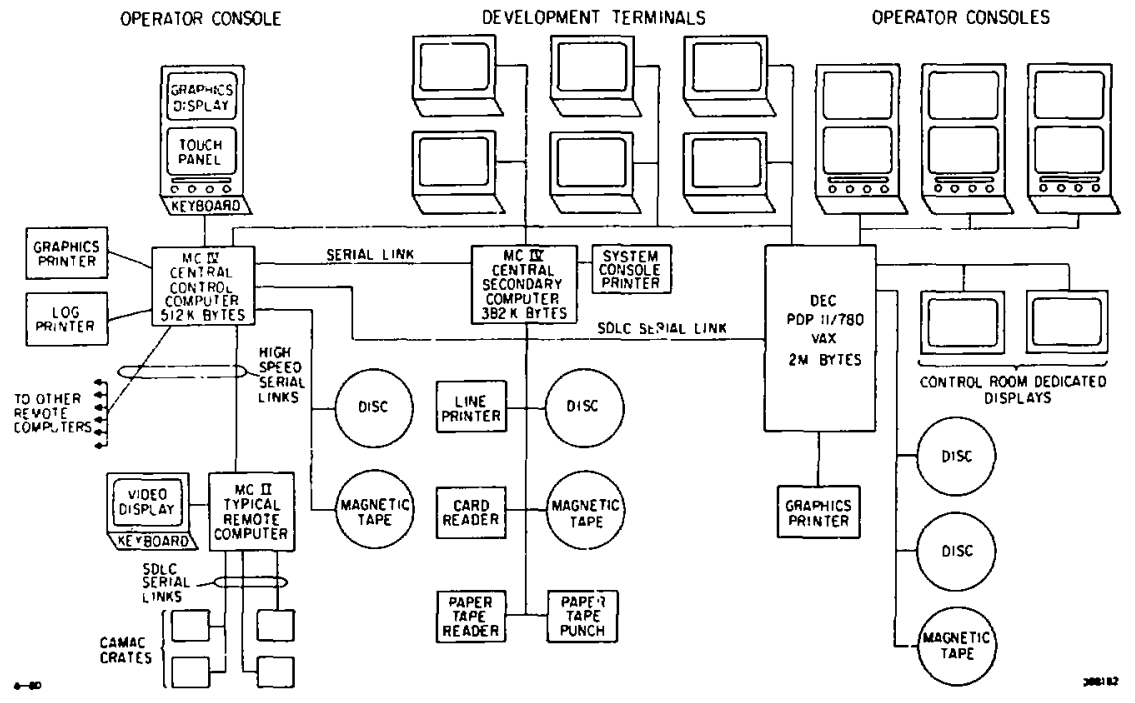

Fig. 3. PEP Computer Control System. 
nous Data Link Control (SDLC) t) technology. A similar link is used to :onnect the MCIV central control computer to the VAX.

The addition of the Vax computer to the system is a departure from earlier planned implementations ${ }^{2}$ ). For this reason, the system is presently in a state of flux with respect to the functions provided by the vax and the two central MCIV computers. The system is evolving towards a configuration that will optimize the use of the strongest features of both the ModComp and VAX software operating systems: the MCIV provides a fast and flexible I/O structure and fast task switching; the VAX provides an operating system that can simultaneously support many physically large operating programs and online users, and provides an environment for fast, efficient program development and maintenance.

Within the next year, the system will evolve to the state where the MCIV central control computer will serve to "download" the remote processors and support the fundamentil I/O data acquisition and control tasks. It will also be used to communicate with the operators through a single console to provide minimal control and monitoring functions in the event of a VAX hardware failure. The VAX computer will provide operator communication through three consoles and will perform essentially all of the higher level monitoring and control functions required for flexible and efficient operation of the ring. The VAX will communicate with the CAMAC crates through the MCIV central control computer. The central secondary MClV will serve as a hardware backup to the central control MCIV.

A remote MCII computer is located in each of the seven IदC sur rort areas. The eighth MClI is used as a spare and for program development. Presentiv, these computers are primarily used as data concentrators for the central computers. As the operational requirements of the ring become more and more demanding, it is expected that more local control and monitoring tasks will be implemented with the remote computers.

\subsection{1nterface and Operator Console Hardware}

The remote computers are interfaced to the ring derices through a high speed CAMAC system which provides a serial communication link that uses the Direct Memory Access (DMA) capability of the MCII's. The CAMAC crates are physically located near the equipment to which they are cennected, and are located as far as $500 \mathrm{~m}$ from the nearest MCII. Most interface requirements for the $\sim 10,000$ signals in the system are satisfied by five basic CAMAC modules; a 16-channel 12-bit analog monitoring module; a 8-channel 12-bit analog control module; a 16-channel latched relay module; a 16 -channel pulsed relay module; and a 16-channel opto-isolated digital monitoring module. Figure 4 shows a profile view of a typical CAMAC crate in the system.

Microprocessor based controllers for the CAMAC crates and MCIl SDLC serial link controllers have been developed for PEP $\left.{ }^{3}, 4\right)$. Tasks in remote computers communicate with the crate controllers by means of messages 

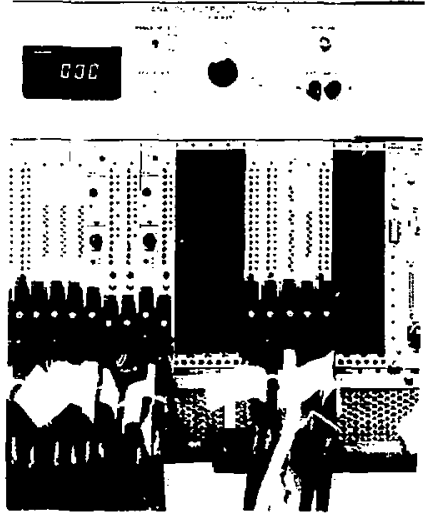

Fis. a. Typical CaMAC crate containing an assortment of Jifital and and log $1 / 0$ modules. The vightmost two slots contajn a Mir slill sute tontrolle?

4 incrementally encoled general purpose slek knols, cach with its own 1 s charac ter plasma tisplay. The operater can perfor the sollowing functions through the ase or the touch panels: the selec. tion of displiys to be presented on the (E) 's: the assignment of slew knohs to the set point control of specirie signals; the bimary control (onioff, in/out, etc.) of specifjc signals; the initiation of contral algorithms; and the selection of other touch panels. Figure 5 shows two adjacent MCIV and VAX operator consoles. describing CAMAC actions to be performed. The crate controllers are designed to quickly execute lists of random CAMAC commands. By using these features, it is pussible to collect input data or to provide output data at the rate of $3 \mathrm{~ms}$ of overhead for the message transaction plus to us per CAMAC conmand.

lach of the operator consoles in the system contains the following equip. ment: a 512 clement by 256 line ejghtcolor full-graphics raster.scan CRT display; a $512 \times 512$ monochrome [ullgraphics raster-scan CRT display with an integral touch panel having the capability of providing an 8 by 8 matrix of to: - ?. buttons; an alpha-numeric keyboard; and

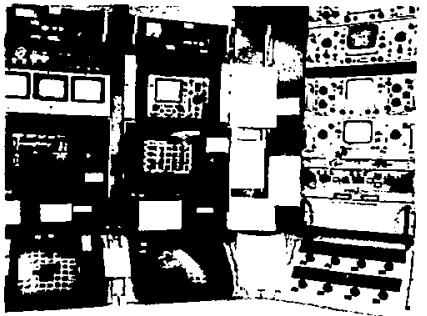

Fig. 5. Two operator consoles. The leftmost touch pancl and display, and the topmost knob pancl are connected to the MCIV central control computer. The remaining elements are connected to the DEC VAX.

\subsection{Applications Surport Softharc}

The routines described here provide a systematic and convenient methou for FORTRAN application programs to communicate with the ring operators, to perform data I/O functions, and to communicate with other applications programs.

\subsubsection{Tata base routines}

The PEP data base ro i ines provide a means for application programs to be written without detailed knowledge about the memsiy or CAMAC location of signals of interest, the units associated with the signals, or their conversion factors. Instead this information is maintained on a common disk oriented data base which provides one disk record for each signal in the pEP 
system. The creation of this data base begins with a trce-structured description of the approximately 13,000 hardware and software data signals. The following excerpt from that description defines 1080 signals associated with vacuum ion-pump power supply chassis:

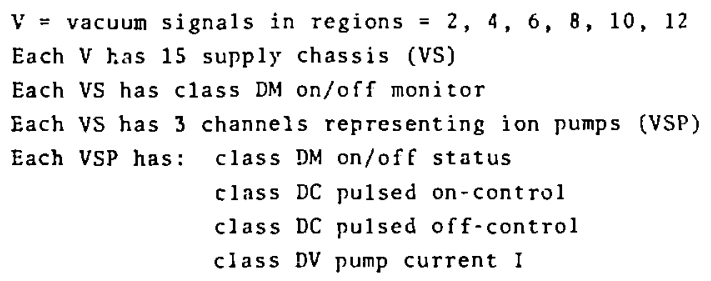

A computer program expands the tree description and assigns a unique signal name and disk record to each signal. The name is formed by a combination of letter: and indices. For instance, "V6S2P3/DC1" refers to the first digital control (DC) signal for the third pump channel in the sccond chassis connected to the region 6 remote computer.

A group of signals may be named by omitting indices. For example, V65P/DC1 refers to the first de signal for all pumps in region 6 and VSP,'DC1 refers the first dc signal for all pumps in the ring. The structured signal names provide an efficient way for programs to access data, but because they are not sufficiently mnemonic for operator use, a "display name" is provided as a data base attribute for each signal.

An important criterion for any large signal data base is that the data for signal names can be quickly located. This is accomplished by preprocessing the tree-structured signal list to form tables and lists which can be used at run time to quickly compute the locations of signal data.

\subsubsection{Data acquisition and control routines}

Data monitoring functions for the ring are performed by a program with an initialization phase that searches through the disk data to form lists of CAMAC commands associated with all of the monitored signals for cach CMMAC crate in the system. These named lists ar" transferred to rheir respective CAMAC rate controllers. In normal operation, the remote computers continuous ly collect data from tireir respective CAMAC crates and forward the refreshed data to the central control computer. The central control computer maintains a copy of the latest data for each signal in its core memory. Applications tasks can access data by providing signals identifiers (SID's) which are offset pointers into the data arrays in the central control computers. A subroutine is provided that quickly converts user oriented signal names to SID's using tables created by the tree-structured signal list preprocessor.

Applications programs can output data to digital CAlAC modules by supplying SID's and data to the data I/O routines. That information is then 
transmitted to the remote computers which use the SID's to find the appropriate CAMAC commands from previously initialized tables. The remote computers then send messages to the appropriate crate controllers which execute the CAMAC commands.

There are two forms of analog control available to applications programs, direct and ramped. For direct analog control ( $A C)$, the applications prograns supply the SID's and data values. This information is transmitted to the remote computer which updates the data values in a table contained in its memory and then sends the table of values to the AC CMMAC moduIes.

Ramped analog control is used to change a group of setpoints simultaneously. The user supplies the end point value of each signal to be changed and a maximum step size. The system computes an increment value for each signal which will cause all signals to reach their end-point values after the same number of steps. A common interrupt pulser is used to synchronize ramping activity in all remote computers.

\subsubsection{Touchpane] routines}

The director program provides application program communications with the PEP operators through the touch panels and slew knobs, and provides communication between programs. The stiucture and operation of the touch paneis is defined by an object code created by a touch panel compiler. This compiler allows the specifications of the location of a button, its title, and the actions to be taken when it is touched by an operator. The following actions may be defined: a specified program may be initiated or terminated; specified dat may be sent to a specified signal; a slew knob may be attached to a signal; an "event" may be decleared which can be uscd to notify programs that the button has been activated. The Director allows any program to simulate operator actions and it also provides a flexible method for program to program communications by allowing saveral options for enqueing/dequeing arbitrarily named messages to/frum a common message pool.

\subsubsection{Graphics routines}

Primary graphics support for the full graphics CRT's has been provided by modifying the Unified Graphics ${ }^{5}$ and Handypak ${ }^{5}$ packages, originally written for the SLAC "Triplex" central computing facility, for use on the MCIV and VAX in conjection with the PEP graphics hardware. Having graphics "calls" compatible with the Triplex system has proven useful because many applications programs have been developed on the Triplex and then moved to the PEP system by use of a RS-232 serial link.

3. CONTROL AND MONITORING OF THE MAGNET POWER SUPPLY, RF, AND VACUUM SYSTEMS

\subsection{Power Supply Control and Monitoring}

There are approximately 25 main chopper power supplies and 150 trim and steering supplies in PEP. The chopper supplies require high-precision control and monitoring, so their analog set-point is determined by 16-bit DAC's 
located within the power supply controllers and their supply current is monitored with high precision transductors attached to a relay-multiplexed integrating digital voltmeter (DWM) system. The trim and steering supplies are interfaced to the computer through standard 12 -bit resolution analog output and analog input CAMAC modules.

Control and monitoring software has been written to allow operators to perform the following functions:

- Display the setpoint and monitored current for each supply in amps and display the operational state of the supplies.

- Attach any supply to a manually controlled knob or ramp any supply to a desired setpoint entered through a terminal.

- Save a set of monitored currents for future use.

- Restore magnet currents to a previously determined set of currents.

- Perform a calibration procedure to determine correction constants to the nominal output current vs DAC setting transfer function.

- Perform a test procedure to test the linearity of the DAC-power supply system.

- "Standardize" the magnet system.

In addition to the relatively straightforward control and monitoring functions described above, an extensive set of on-line lattice modeling and control programs $\left.{ }^{2}{ }^{7-\theta}\right)$ are provided that allow the ramping of power supplies without loss of beam to a new lattice described only in terms of the lollowing beam parameters: the betatron tune $\nu_{x}, v_{y}$; the betatron functions $B_{x}, B_{y}$; the interaction dispersion function 7 ; the chromaticities; and the beam energy E. Magnet strengths are derived from the on-line theorctical mathematical models of the ring and are then passed through polynomial transfer functions for magnetic measurement and transductor calibrations in order to obtain the required magnet currents.

\subsection{RF System Control and Monitoring}

Typically, RF systems for storage rings are very large and complex, and PEP is no exception. Approximately 3000 computer signals are related to the control and monitoring of power supplies, phase controls, thermocouples, tuner positions, etc., that are associated with the 12 PEP RF stations. Up to the present time, most of the RF programs have been of the "look and adjust" type. Further software efforts will center on providing extensive surveillance programs. Also, fully automated routines will be provided that set up a group of stations for a specified operating configuration and will automatically adjust $R F$ controls to maintain a constant synchrotron tune $v_{S}$ while the magnetic lattice is ramped between configurations. 


\subsection{Vacuum System Control and Monitoring}

Approximately 250 channels of vacuum ion-pump power supplies are used for the ring. Each channel provides $5 \mathrm{kV}$ to an open circuit and $50 \mathrm{~mA}$ of current to a short circuit. The current provided by each channel is monitored by the computer system over $\mathrm{j}$ ts range of interest - $1 \mathrm{\mu A}$ to $10 \mathrm{~mA}$ - - by the relay-multiplexed DVM system. Individual ion-pump current readouts are used to provide coarse information concerning the ring vacuum. Approximately 100 ion-gauges are interfaced to the computer to provide more accurate information for speciric geographic regions.

A system to help pinpoint the location of a catastrophic vacuum failure has been provided, but is not yet operational. This system utilizes a cAMAC module to continually scan "overcurrent" outputs from all ion-pump channels in a given region. The time of the last transition to the "overcurrent" stutc is logged into the module. Then after the failure, the computer system proiluces a record of the sequence of pump overcurrent readings. This sequence can be used in determining the location of the vacuum fault.

\section{BLAM MOAITORS}

\subsection{Beam Position Monitoring System}

Figure o shows a block diagram of the beam position monjtoring equipment. Bi-polar pulses from \& buttons associated with 18 monitors in each region are multiplexed with coaxial relays to provide signals to a single detector system ${ }^{19}$. This detector system jinearly stretches and integrates the pulses to prowise a broal $20 \mathrm{~ns}$ pilso. A computer controlled CANA timing modile provides a reference to a high speed synchronous sampling circuit that creates a de roliage directly related to the pejk of the input pulse.

Upon a command from the operator, 72 de voltages (18 monitors $\times 4$ buttons) are read by the local remoce computer and passed to the central computer. Horizontal and vertical bean positjons are then calculated by using the button values. Arter the scan has been compicted, a display showing the orbit is

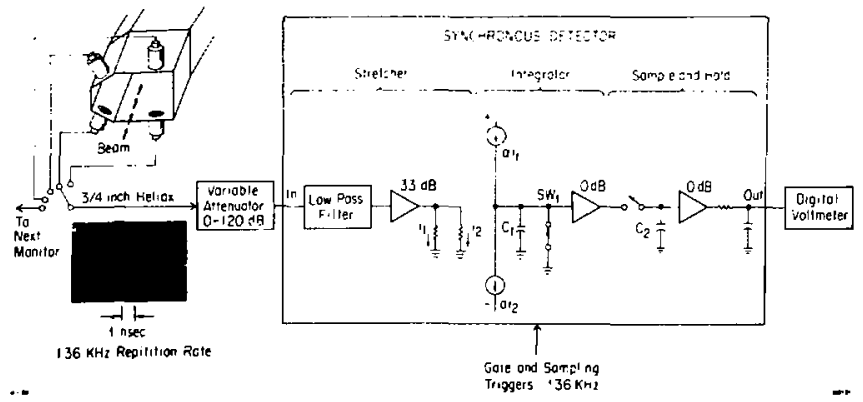

Fig. 6. Beam position monitoring system block diagram. 
presented to the opesator. The operator can then initiate a program to calculate magnet steering currents for global or regional orbit corrections"1). The predicteg "corrected" orbit is calculated and is then superimposed on the orbit scan display for the operator's approval. The operator can ilen reject the correction or initiate the correction process to ramp the stecting supplies to their new values. The whole process of making the pon is ion measurement and performing the orbit correction requires $5-11$ mimules.

Because of their extreme importance to the sucessful ogerition ef : lle

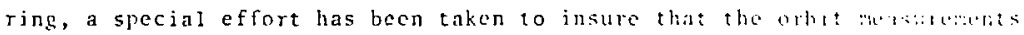

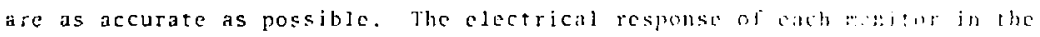
system was measured before its chamber was anstalled. A presisinn lig developed for this purpose inserted a $30 \mathrm{~cm}$ transmitting antenna into the ond of the chamber. The responsc of the buttons was measured for several transverse antenna positions. This information was then uscd to derive lienitorspecific constants for the on-line position calculations polynomials. Aignment data is also factored into the calculations to correct for small positional installation errors.

\subsection{Synchrotron Iight System}

The synchrotron 1 ight emitted from the bending electron and positron beams at PEP are used to determine many important heam parameters related to beam shape and intensity.

Two thin beryllium mirrors, one for $e^{+}$and onc for thears, are iocated within the bean chamber. They reflect visible light theupl al 17 alth, consisting of a quartz window and a series of mirrors, to a small surface building. The surface building houses a light table that supports a system of light splitters and scnsors used to perform the following measurements.

- A portion of the light is focussed directly on TV cameras for direct viewing by operators.

- A portion of the $1 \mathrm{ight}$ is focussed on photodiodes to provide a mcasurement of the total current for each beam, and to provide individual bunch current measurements.

- The horizontal and vertical profile of each beam is displayed on CRT's whose input is derived by photomultipliers sensing a portion of light reflected by oscillating mirror scamers.

- A high speed (100 ps) photodiode and sampling oscillescope are used to examine the length of a single bunch. The light for this measurement is delayed with a $50 \mathrm{~m}$ optical delay path to accommodate the use of a beam derived trigger for the sampling scope.

\subsection{Tune Measurement}

The betation tune of the bean is measured by transversely exciting the beam with excitation amplifiers. Fast pulses received from striplines are 
demodulated and analyzed with a low-frequency wave analyzer. The synchrotron tune is obtained by phase-modulating an RF klystron and measuring the frequency of resultant transverse motion with the wave analyzer.

\subsection{Transverse Beam Feedback System}

Figure 7 shows the biock diagram of a closed-loop (ecdback system ${ }^{12}$ ) that has been developed Cor PEP to damp transvirse oscillations of the beam. Twelve wide-band detectors ${ }^{3}$ ) are used to sample-and-hold vertjcal and horizontal "error" signals lor the 3 bunches in each beam. A modulated suppressed-carrier $9.8 \mathrm{MHz}$ excitation amplifier is used to drive the beam and close the loop. The excitation amplifier is modulated by a complex timing and multiplexing system that selects the appropriate horizontal and vertical detected "e.ror" signals to apply to a bunch as it passes by the excitation electrodes.

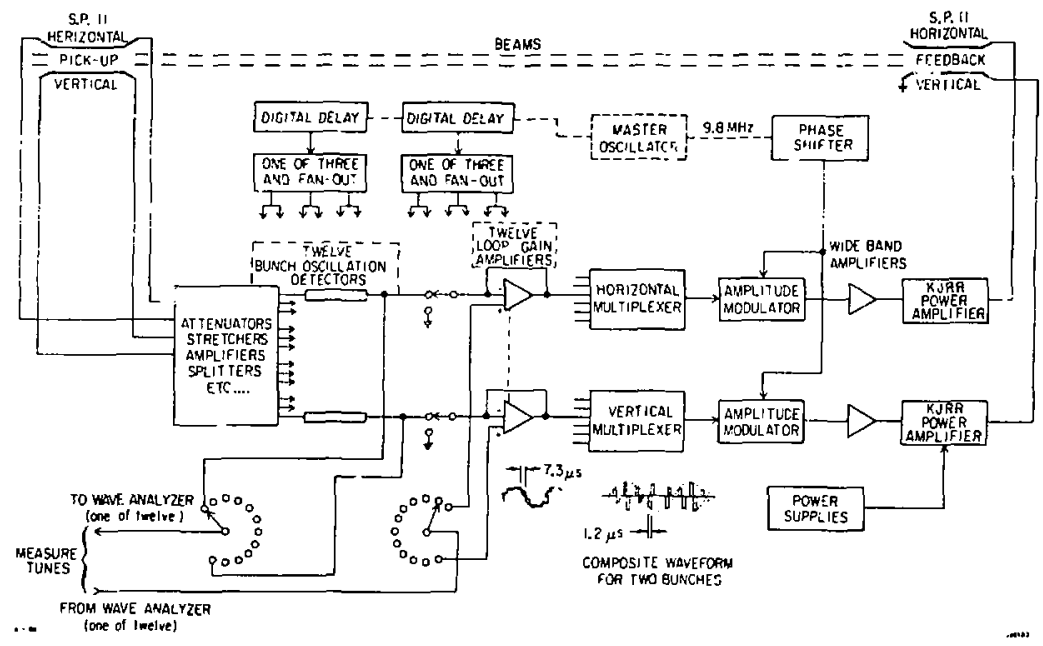

Fig. " . Transverse beam feedback system block diagram. 


\subsection{Direct Current Current Transformer (DCCT)}

A DCCT is provided to obtain the total current in the ring. The DCCT is primarily used as a calibration device for other beam intensity measurement devices which have no means of absolute calibration. A non-conducting ceramic gap is placed in the be iline to prevent the measurement of low frequency lenkage currents and current induced into the beanline as a result of magnet ramping.

5. TIMING, COMMUNICATION, AND PERSONNEL PROTECTION SYSTEMS

\subsection{Timing System}

The PEP timing system generates gates and timing pulses for use by all beam sensors and exciters. The system supports modules that produce timing signals synchrotnous to the beam and allow for manual or computerized phase adjustment in $2.7 \mathrm{~ns}$ (1 RF bucket) steps. The system also provides complex tring patterns used by the SLAC LINAC during the injection process.

\subsection{Communication}

Quality voice communication has proven to be one of the most vital omponents in the checkout and maintenance of PEP equipment. Ml main equipment and control areas at PEP have been connected by a system of highfidelity intercoms. Additionally, 20012 -channel headset intercom stations have been located in the beam tunnel and near all equipment racks. This system allows convenient communication between any two pieces of equipment on the PEP site.

\subsection{Personnel Protection System}

The physical layout of the ring requires that six experimental areas be shut down if access into any experimental area is desired. Becausc of this fact and the fact that emergency repair entries into the tunnel provide major disruptions in a physics program, extensive efforts have been made to provide a Personnel Protection System (PPS) that minimizes the downtime cicated by entries into protected areas without sacrificing the overall safaty aspects of the ring. The ring and its injection lines have been divided into 14 individually monitored and controlled zones. "y carefully monitoring and controlling personnel accesses to specific zones, an operator can switfty and safely resecure the ring after the entry by securring only those specific zones that were compromised.

A prototype automated entry system is presently undergoing tests. This system maintains records of entries and exits to/from an area that is in "controlled" access. A "bar-code" strip, similar to those used in retail stores, has been attached to the desimeters of all SLAC personnel. An inexpensive reader is used at the entry gate to transmit the coded data via a RS-232 message to a micro-processor system in PCR. 


\section{ACXNOWLEDGEMENTS}

We wish to acknowledge the efforts of all the people associated with the specification, development, construction, installation and checkout of the PEP Instrumentation and Control System. The success of the system is a function of the hard work and dedicated perfermance of literally hundreds of people associated with all aspects of the project.

\section{REFERENCES}

1) J. R. Kersey, Synchronous Data Link Control, Data Communicatious, McGraw-Hi11 Publications, 49-60 (May/June, 1974).

2) A. Chao et al., PEP Computet Control System, lI:E Ttans. Nucl. Sci. NS-26, 3268-3271 (1979).

3) A. Altmann and R. Rozzano, ModComp to SDLC Link Controller, PEP internal document.

4) A. Altmann, R. Belshe, R. Dwinell, J. Fox and N, Spencet, CAMAC Mictoprocessor Crate Controller, Revision J, PEP internal document.

5) R. C. Beach, The SLAC Unified Graphics System Programming Manua1, Stanford Linear Accelerator Center Report rGTMH170 (1975).

6) C. A. $\operatorname{Logg}$, A. M. Boyarski, A. J. Cook and R. L. A. Cottrell, DPAK and HPAK-A Vrrsatile Display and Histogram Package, Stanford Linear Accelerator Center Report SLAC-156 (1976).

7) A. S. King and M. J. Lee, Stanford Linear Accelerator Center internal note PEP-262.

8) 1. H. R. Donald, P. L. Morton and H. Wiedemann, Chromaticity Correction in Large Storage Rings, IEEE Trans. Nucl. Sci. NS-24, 1200-1202 (1977).

9) 11. Wiedemann, Stanford Linear Accelerator Center internal note PEP-220.

10) J.-I. Pellegrin, Synchronous Detection of Beam lr luced pulses, Stanford Lineat Actelerator Center Reprot SLAC-PUB-2359 (1979).

11) E. Close, M. Cornacchia, A, 5. King and $M$. J. Lec, A Proposed Orbit and Vertical Correction System for PEP, IEEE Trans. Nucl. Sci. NG-26, $3502-5.304(1979)$.

12) J.-L. Pellegrin and J.R. Rees, Beam Excitation and Damping with the Traisverse Feedback System, Stanford Linear Accelerator Center internal note PEP-315 (1979).

13) J.-L. Pellegrin, A Normalized Detector of Beam Transverse Oscillations, Stanford i inear'Accelerator Center Report SLAC-PUB-3209 (1979). 